\title{
REPRODUÇÃO APÓS OS 30 ANOS \\ NO ESTADO DE SÃO PAULO
}

\author{
ELZA SALVATORI BERQUÓ \\ BERNADETTE CUNHA WALDVOGEL \\ SANDRA GARCIA \\ Carlos Eugenio de Carvalho Ferreira \\ TANIA DI GIACOMO DO LAGO \\ LUÍS EDUARDO BATISTA ${ }^{1}$
}

\section{RESUMO}

$\mathrm{O}$ artigo se propôs investigar o adiamento da reprodução no estado de São Paulo, entendido como postergação do nascimento do primeiro filho, de mulheres maiores de 30 anos. Como estratégia metodológica, utilizou-se abordagem por período e por coortes. Dados sobre nascimentos provieram do Registro Civil. Os Censos forneceram os efetivos populacionais femininos por idade, ao longo do período analisado (1960 a 2010). Verificou-se que na coorte de 1980 a fecundidade do grupo 30-39 passou a ter maior participação na fecundidade total. Entre 2000 e 2010 , cresceu a proporção de mulheres sem filhos em todas as faixas etárias. A fecundidade por ordem de parturição revelou aumento da proporção de nascimentos de primeiros filhos entre mulheres de 30 a 39 anos, sugerindo a existência de adiamento, temporário ou definitivo.

PALAVRAS-CHAVE: reprodução; adiamento do primeiro filho; abordagem por coortes e período.

\section{ABSTRACT}

This paper provides an investigation on the delaying of reproduction in the state of São Paulo, which is understood as the postponement of the birth of the first child of women older than 30 years of age. As a methodological strategy, we approached the issue by period and cohorts. Data on births were obtained from the Civil Registry. The Population Censuses provided the effective women's population size by age across the analyzed period (1960-2010). We verified that in the 1980 cohort the 30-39 fertility group increased its share in total fertility. Between 2000 and 2010 , there was an increase in the share of childless women in all age groups. Fertility by birth order of the child showed an increase in the proportion of first-child births among 30-39 year-old women, indicating temporary or permanent postponement.

KEYWORDS: reproduction; postponement of first child; approached by period and cohorts.

[1] Os autores fazem parte do grupo Mulher de Trinta, que há dois anos se reúne no Cebrap para troca livre de ideias no campo da demografia.

\section{INTRODUÇÃo}

Estudos sobre a transição demográfica em países europeus têm registrado o declínio constante das taxas de fecundidade a partir dos anos 1970. Esses estudos têm apontado o uso de métodos contraceptivos mais seguros e mudanças nos padrões comportamentais, fatores constituintes dessa transição (Lesthaeghe e Neidert, 2006; Lesthaeghe, 2010). 
Métodos contraceptivos têm sido usados desde há muito tempo, porém a pílula contraceptiva, descoberta ocorrida há mais de cinquenta anos, pode ser considerada o avanço mais importante para o controle da reprodução, por ser de uso fácil, confiável e controlado pela mulher. $\mathrm{O}$ uso disseminado da pílula, associado a um contexto de mudanças de valores sociais, culturais e comportamentais, possibilitou transformações no comportamento reprodutivo, com consequente impacto na estrutura etária da fecundidade.

De fato, dentre as mudanças demográficas mais importantes das últimas décadas, destaca-se a elevação da idade da mãe ao nascimento do primeiro filho. Estudos populacionais têm identificado fatores socioeconômicos, tais como maiores níveis de renda e maior grau de escolaridade, como os principais impulsionadores dessa tendência crescente na Europa (Beets, 2008; Billari, Liefbroer \& Philipov, 2006; Mills et al., 2011; Ní Bhrolcháin \& Beaujouan, 2012).

Vale mencionar que esse fenômeno é também observado nos Estados Unidos. Na realidade, nas últimas três décadas, além de queda da fecundidade, tem havido um aumento crescente de casais sem filhos, um componente importante da baixa fecundidade nesse país. Com dados do U.S.Census Bureau de 2011 , entre 1976 e 2010 , o percentual de mulheres sem filhos na faixa etária de 35 a 39 anos passou de $10,5 \%$ para $19,7 \%$, enquanto na faixa etária de 40 a 44 anos foi de 10,2\% para $18,8 \%$, respectivamente (Tunalilar \& White, 2012 ).

Além dos elementos explicativos apontados, há estudos relacionando períodos recessivos como fortes componentes associados à baixa fecundidade apresentada pelos EUA e Europa, em anos mais recentes (Sobotka et al., 2011).

No Brasil, esse processo de transformação, apesar de desigual e regionalmente diverso, tem-se caracterizado pela maior participação da mulher no mercado de trabalho, no aumento da escolaridade feminina e nas redefinições das relações de gênero. Muito embora esse processo tenha ritmos eespecificidades próprios, há de se registrar, em regiões metropolitanas, o fenômeno do adiamento da maternidade para após os 30 anos de idade (Wong \& Perpétuo, 2009).

Em nosso meio, embora ainda não se disponha de estudos demográficos detalhados sobre a reprodução tardia, há indícios de que ela já começa a mostrar sinais de sua presença.

De fato, a análise da distribuição relativa das taxas específicas de fecundidade em relação à fecundidade total revela que a partir dos 30 anos de idade o percentual passou de $27,6 \%$ para $31,3 \%$ entre 2000 e 2010, ou seja, aumento de 3,7 pontos percentuais (Tabela 1).

Esses sinais são mais acentuados entre as mulheres com rendimento nominal mensal familiar per capita superior a 5 salários míni- 


\begin{tabular}{c|c|c} 
Grupos etários & 2000 & 2010 \\
$15-19$ & 18,8 & 17,7 \\
\hline $20-224$ & 29,2 & 27 \\
\hline $25-29$ & 24,4 & 24 \\
\hline 30 ou + & 27,6 & 31,3 \\
\hline Total & 100 & 100
\end{tabular}

Fonte: IBGE, Censos Demográficos 2000 e 2010.

mos em 2000 , quando a fecundidade correspondente à faixa etária de 30 a 34 anos representava $32,5 \%$ da fecundidade total. O processo de adiamento da fecundidade entre as mulheres com maior poder aquisitivo continuou de forma mais acentuada em 2010 , quando essa curva se desloca ainda mais para a direita, destacando-se das demais faixas de renda, como mostra o Gráfico 1.

A reprodução tardia via adiamento pode ser apreciada, também, analisando-se os dados da Pesquisa Nacional de Demografia e Saúde(PNDS) de 1996 (Benfam) e 2006 (Brasil), que apontam um aumento no percentual de mulheres de 30 anos ou mais de idade que ainda não tinham tido filhos até aquele momento, que passou de 9,9\% para 11,1\%. Vale notar ainda que, em $2006,8 \%$ das mulheres tiveram o primeiro filho após os 30 anos de idade (Berquó \& Garcia, 2012).

No estado de São Paulo também existem evidências de forte queda da fecundidade e de transformação da sua estrutura etária decorrentes de processos de adiamento ou mesmo do encerramento da reprodução. O Censo Demográfico de 2010 indica que a proporção da população feminina "sem filhos" aumentou sensivelmente em todas as faixas etárias, em relação aos dados do Censo de 2000 (Gráfico 2).

Tais elementos suscitaram uma análise mais acurada da evolução da fecundidade nesse estado, com ênfase no comportamento reprodutivo após os 30 anos de idade.

\section{OBJETIVO}

Este artigo se propõe a estudar a tendência da fecundidade no estado de São Paulo, nos últimos cinquenta anos, segundo período e geração, para verificar a ocorrência de adiamento da reprodução no grupo de mulheres de 30 anos e mais de idade. 


\section{GRÁFICO I}

Padrão etário da fecundidade segundo classes de rendimento nominal mensal familiar per capita Brasil - 2000 e 2010

2000

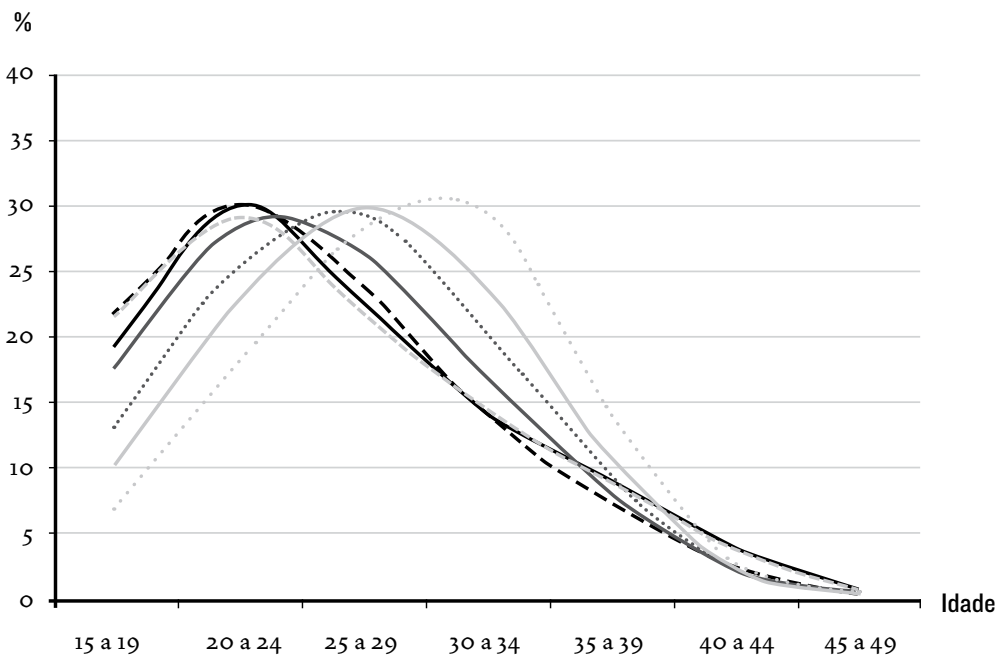

2010

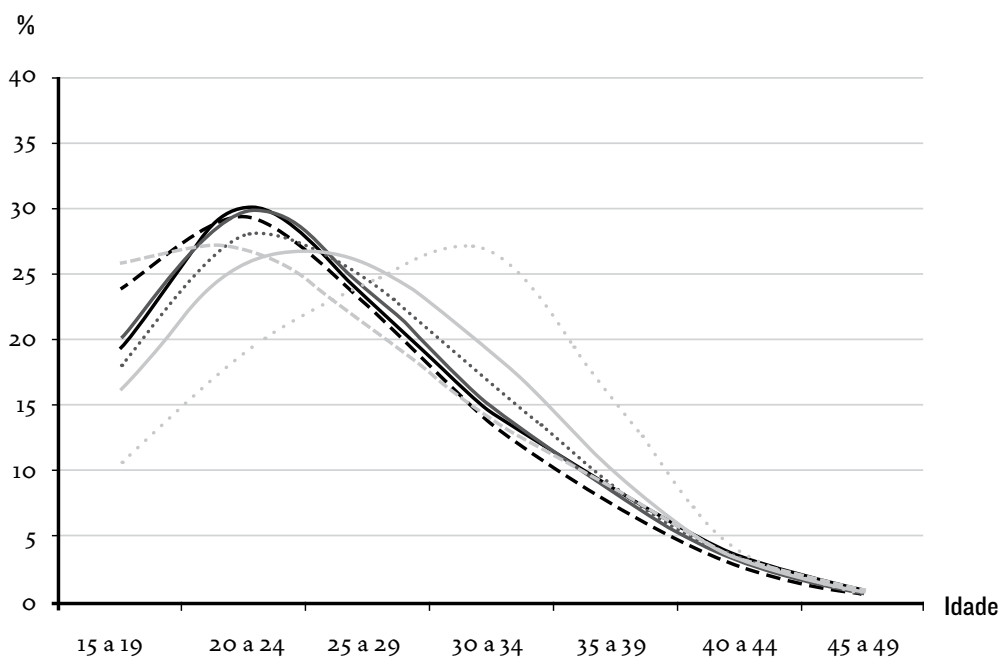

Até $1 / 2$ sal. mín

De 2 a 3 sal. mín.

Sem rendimento

- - De l/2 a l sal. mín.

De 3 a 5 sal. mín.

De I a 2 sal. mín.

Mais de 5 sal. mín.

Fonte: IBGE, Censos Demográficos 2000 e 2010. 


\section{GRÁFICO 2}

Proporção da população feminina sem filhos

Estado de São Paulo - 2000 e 2010

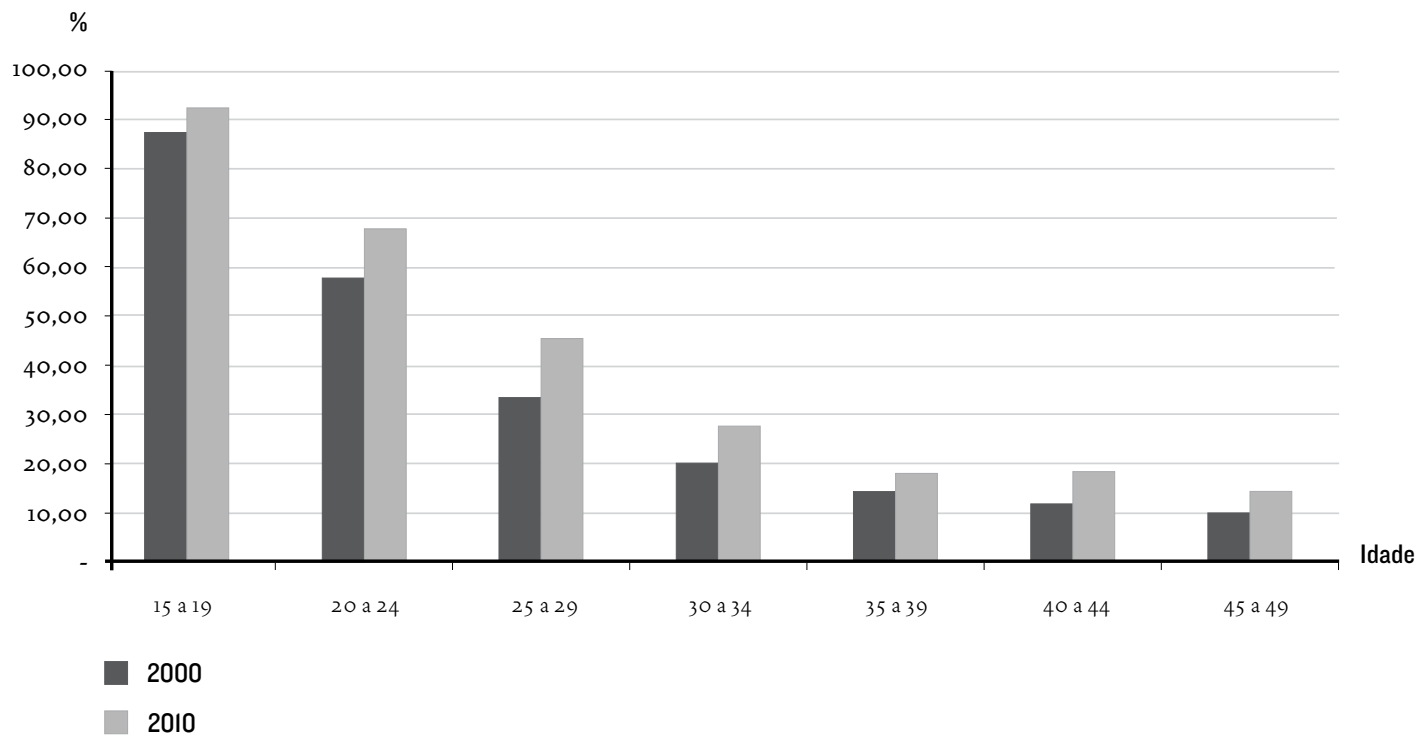

\section{METODOLOGIA E FONTES DE DADOS UTILIZADAS}

Neste artigo, considerou-se "adiamento da reprodução" a postergação do nascimento do primeiro filho.

Como estratégia metodológica, foi selecionado o estado de São Paulo como unidade geográfica de análise e foram identificadas as mulheres na faixa etária de 30 anos e mais de idade que tiveram o primeiro filho após essa idade, ou que ainda não tiveram filhos nascidos vivos, eque, portanto, continuariam adiando temporária ou definitivamente a reprodução.

Os dados básicos utilizados neste estudo são originários de duas fontes clássicas: Censos Demográficos e Registro Civil.

Os dados de nascimentos, classificados segundo idade da mãe, ano de ocorrência e ordem de filiação, foram obtidos do banco de estatísticas vitais da Fundação Seade, que mantém um sistema de coleta mensal nos cartórios de Registro Civil do estado de São Paulo e realiza um confronto contínuo e individualizado, entre as informações do Registro Civil e aquelas da Declaração de Nascimento originárias dos hospitais/maternidades (Waldvogel \& Ferreira, 2003).

As informações censitárias produzidas pelo IBGE possibilitaram obter os efetivos populacionais femininos por idade, para o cálculo das taxas de fecundidade ao longo do período analisado. 
Os indicadores referentes aos anos intermediários terminados em 5 foram calculados com base em estimativas populacionais realizadas pela Fundação Seade. Essas estimativas levaram em consideração o crescimento vegetativo registrado no período e o saldo migratório estimado.

Os Censos Demográficos de 2000 e 2010 viabilizaram as estimativas da fecundidade segundo faixas de renda, que aparecem na introdução deste trabalho. Foram utilizadas as técnicas indiretas (razão $\mathrm{P} / \mathrm{F}$ ) para cada intervalo da categoria considerada.

Para a análise da evolução da fecundidade no estado de São Paulo, no período de 1960 a 2010 , foram consideradas duas abordagens clássicas da análise demográfica, por período e por coorte.

Para efeito de identificação das coortes, optou-se por nomear cada uma delas com o ano correspondente à faixa etária que inicia o período reprodutivo considerado neste trabalho. Dessa forma, a "coorte de 1960 " corresponde às mulheres que, em 1960, pertenciam à faixa etária de 15 a 19 anos.

A comparação dos resultados da aplicação dessas duas perspectivas de análise, que constitui o principal recurso metodológico deste trabalho, possibilita melhor compreensão da evolução recente dos níveis de fecundidade em São Paulo e caracterização das transformações em sua estrutura etária.

Em situação de fecundidade declinante, no contexto da transição demográfica brasileira e mais especificamente no estado de São Paulo (Waldvogel et al., 2003), os indicadores de nível da fecundidade elaborados a partir da classificação dos dados por coorte podem diferir de maneira sensível daqueles calculados a partir da classificação por período.Assim, a comparação dos índices transversais e longitudinais permite trazer mais elementos para a compreensão das mudanças de nível e distribuição etária da fecundidade.

A idade média da fecundidade foi o indicador sintético utilizado para medir as mudanças ocorridas na distribuição etária da fecundidade e consiste na média ponderada das idades das mulheres, tendo como fator de ponderação, a cada faixa etária, a taxa de fecundidade correspondente do período ou da coorte.

\section{ESTUDO DA FECUNDIDADE POR PERIODO}

E POR COORTE NO ESTADO dE SÃO PAULO DE 1960 A 2010

\subsection{Queda nos niveis da fecundidade}

As taxas de fecundidade da mulher paulista vêm se reduzindo desde a segunda metade do século passado em contínua e rápida queda, ficando inferiores ao nível de reposição no inicio da primeira década do século XXI, como se observa no Gráfico 3. 


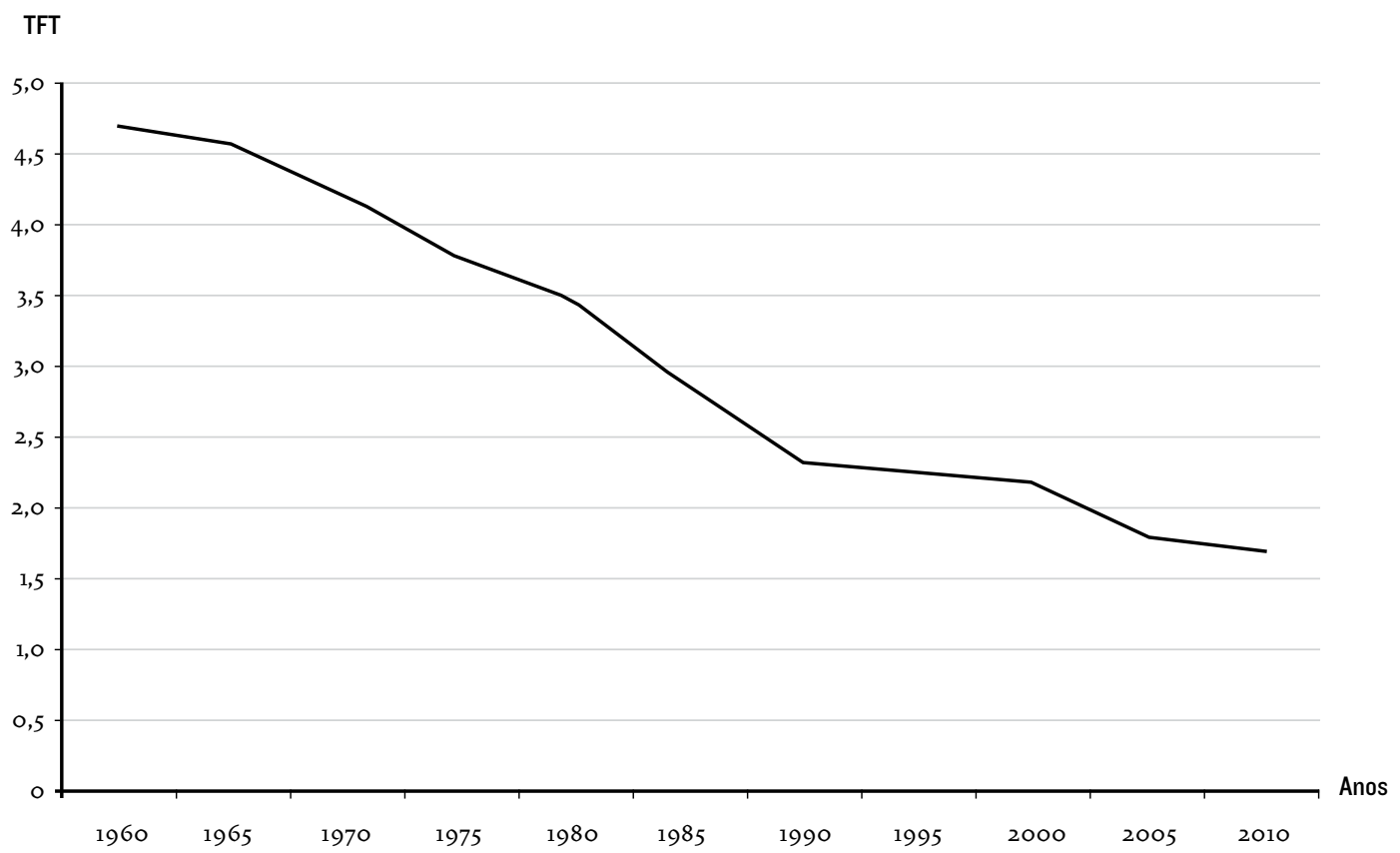

Elaborada com base nas informações do Registro Civil, a taxa de fecundidade do estado de São Paulo passou de 4,7 filhos por mulher, em 1960, para 1,7 em 2010. Em vinte anos, houve redução de quase dois filhos por mulher, quando a fecundidade passou de 4,2 filhos em 1970, para 3,4 em 1980, e 2,3 em 1990. Nos anos 1990, os níveis da fecundidade quase não se alteraram, porém, durante a década seguinteo ritmo de decréscimo se acentua e atinge metade do patamar observado nos anos 1980 (Yazaki, 2011 e 2013; Waldvogel et al., 2012).

O comportamento da fecundidade alterou consideravelmente a evolução do número de nascidos vivos no estado de São Paulo. Observa-se que, em 1960, nasceram 437 mil crianças, registrando-se tendência crescente até o ano de 1982, quando ocorreu o maior volume, com 772 mil nascimentos. Em 2010 esse volume foi de $602 \mathrm{mil}$, ou seja, redução de 170 mil crianças nascidas no estado quando comparado ao total do ano de pico.

A tendência observada no volume anual de nascimentos no estado de São Paulo é determinante na formação das novas gerações da população paulista. A fecundidade declinante associada a gerações femininas cada vez menores intensificará o decréscimo do número de nascimentos. 
4.2. Evolução das taxas de fecundidade para cada grupo quinquenal de idade

Entre 1960 e 2010 , observa-se que o padrão etário da fecundidade da mulher paulista alterou-se sensivelmente, como mostra o Gráfico 4.

Nas três primeiras décadas analisadas, a queda da taxa de fecundidade total esteve associada à importante redução das taxas específicas de todos os grupos etários, com exceção das idades entre 15 e 19 anos, que oscilaram em torno de um mesmo patamar relativamente reduzido.

Entre 1990 e 2000 , observa-se redução nas taxas especificas de fecundidade entre as mulheres de 20 a 29 anos e manutenção dos níveis acima de 30 anos. Já na última década analisada, a redução das taxas específicas envolveu também o grupo de 15 a 19 anos. Importante salientar o aumento verificado para as mulheres de mais de 30 anos entre 2005 e 2010: no grupo etário de 30 a 34 anos, a taxa específica de fecundidade passou de 0,0670 para 0,0684; no grupo 35 a 39, de 0,0351 para 0,0378; no grupo de 40 a 44, de 0,0095 para 0,0099; e no de 45 a 49, de 0,0005 para $0,0006$.

\section{GRÁFICO 4}

Taxas de fecundidade, por grupos de idade

Estado de São Paulo - 1960 a 2010

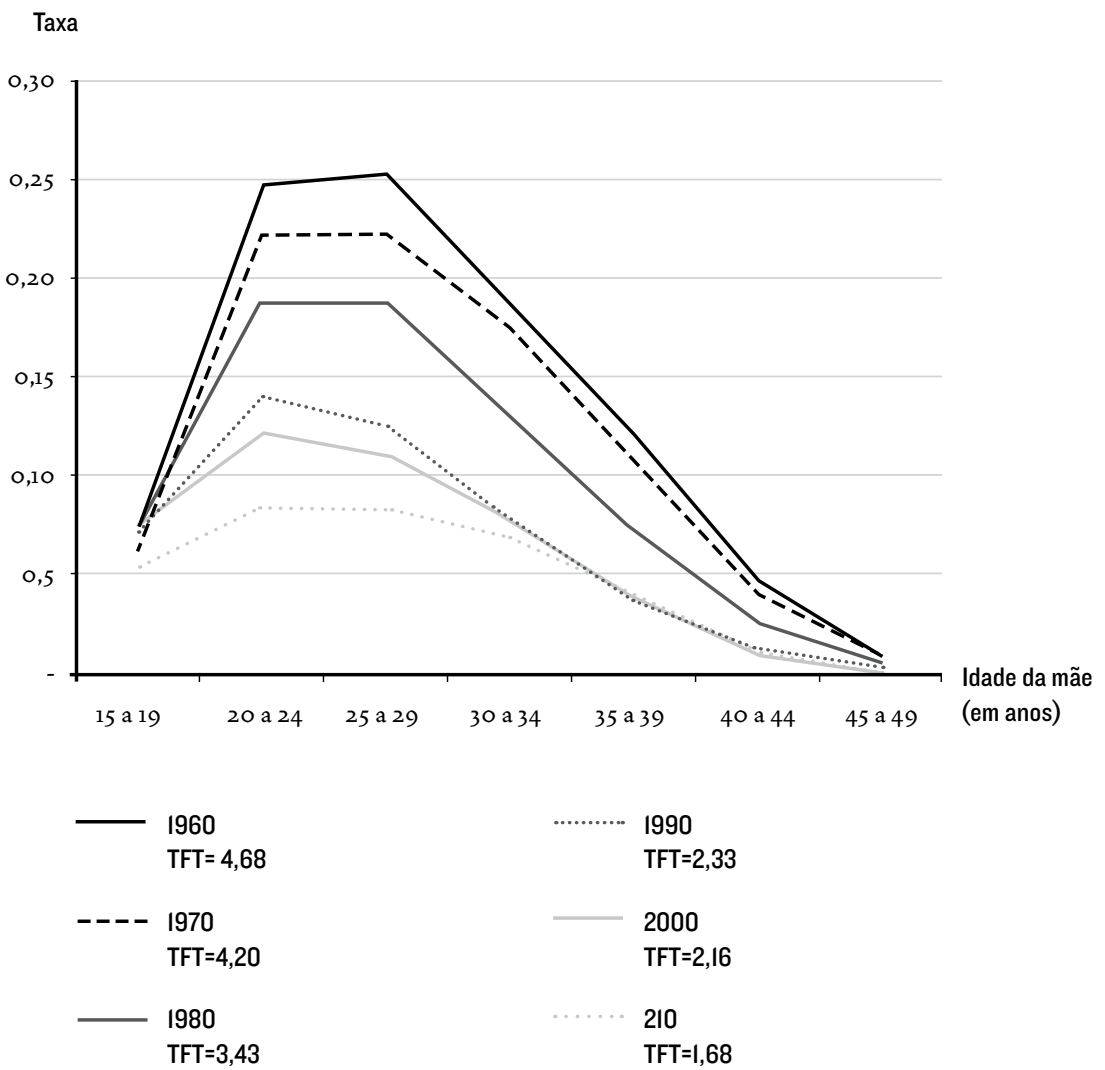


GRÁFICO 5

Participação das taxas de fecundidade das mulheres com menos de 30 anos e de 30 anos e mais na TFT (\%)

Estado de São Paulo - 1960 a 2010

\section{Participação}

(\%)

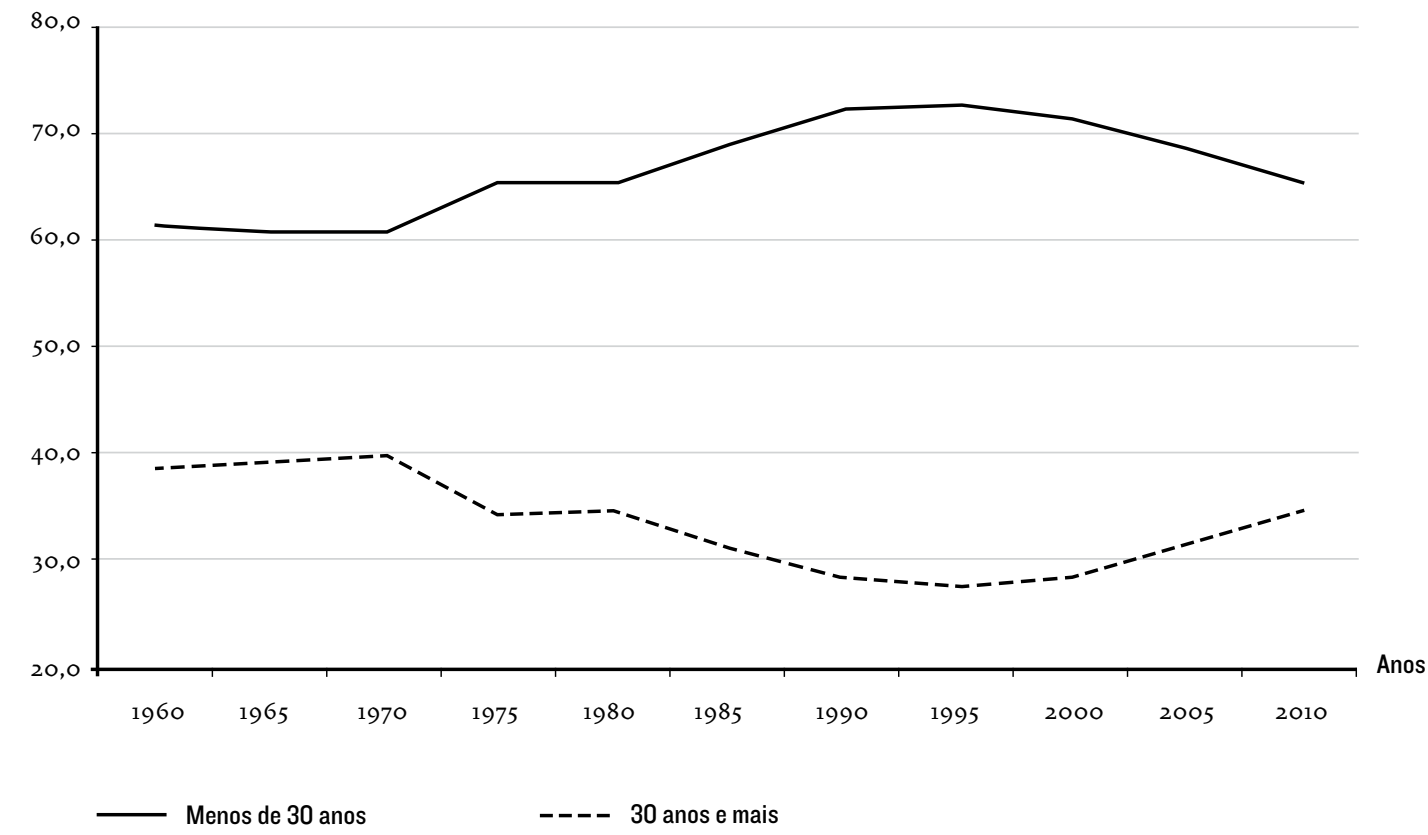

Fonte: Fundação Seade.

O comportamento da queda diferenciada para as taxas específicas de fecundidade resultou, em 2010 , em um padrão etário mais dilatado em relação às curvas dos anos anteriores, que concentravam a fecundidade principalmente entre as mulheres de 20 a 29 anos de idade.

A referida tendência observada provocou transformações profundas no peso relativo da fecundidade de cada grupo etário na fecundidade total, durante os cinquenta anos analisados, como mostra o Gráfico 5 .

Observa-se que, em 1960, a fecundidade das mulheres paulistas com menos de 30 anos participava com $61,4 \%$ da fecundidade total, aumentando para 72,4\% em 1990, quando então passou a diminuir até atingir $65,3 \%$ em 2010.

Por outro lado, as mulheres com 30 anos e mais, que participavam com $38,6 \%$ da fecundidade total em 1960 , reduziram tal participação para $27,6 \%$, em 1990, e a partir daí passaram a aumentá-la, respondendo por $34,7 \%$ da fecundidade total em 2010.

Essas mudanças também se refletem na idade média da fecundidade, como se observa no Gráfico 6. 


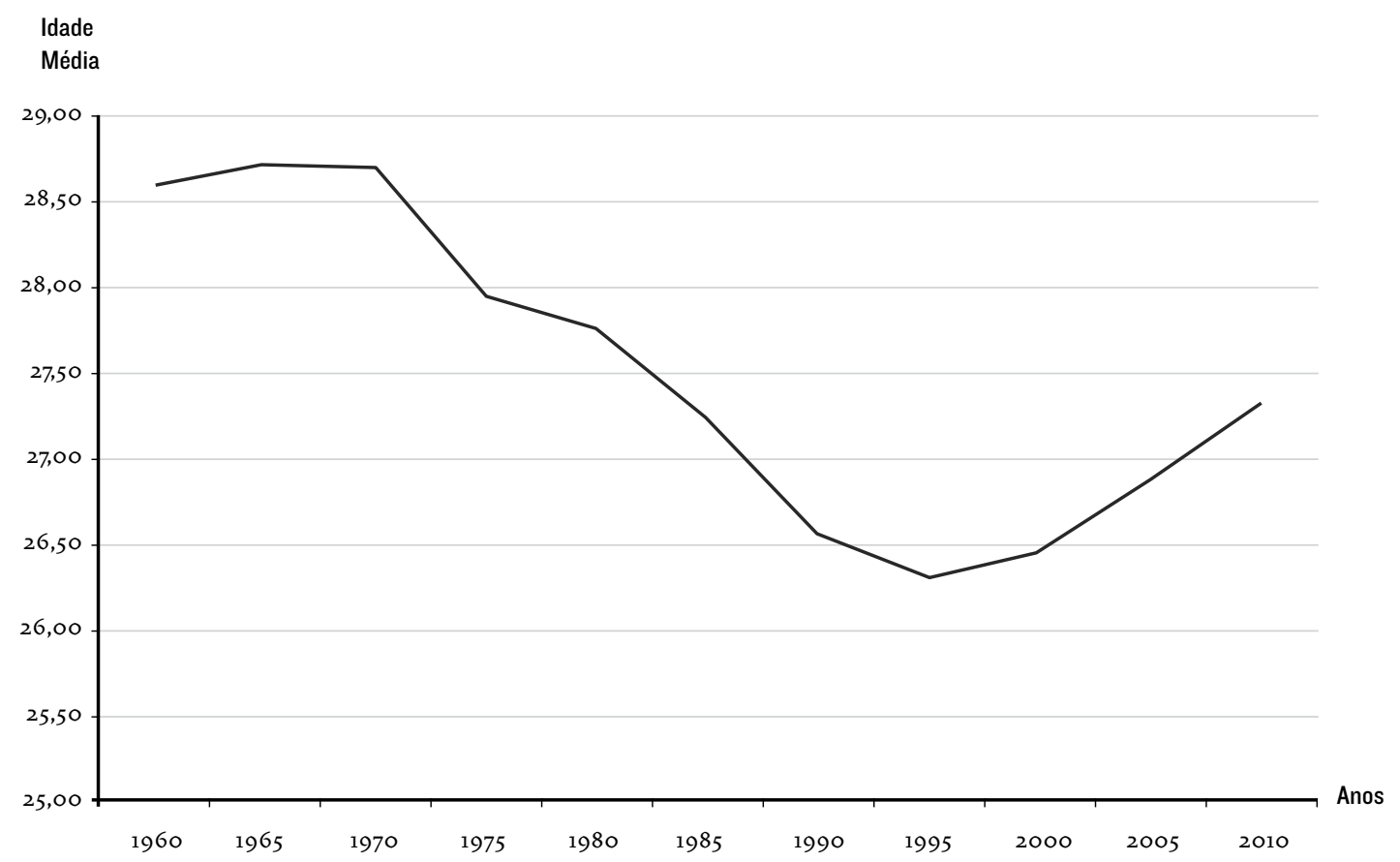

Fonte: Fundação Seade.

Em 1960, a idade média da fecundidade da mulher paulista era de 28,6 anos. Permaneceu nesse patamar até 1970 (28,7 anos) e passou a diminuir sistematicamente até 1995 , quando atingiu 26,3 anos. A partir daí ocorreu reversão na tendência e a idade média da fecundidade passou a crescer, atingindo, em 2010, 27,3 anos.

Vale ressaltar que a tendência recente de aumento da idade média da fecundidade, nos últimos quinze anos, deu-se em período de baixa fecundidade e padrão etário dilatado nas idades superiores a 30 anos, o que pode levar a supor que a mulher paulista está adiando o início da reprodução.

\subsection{Ordem de filiação dos filhos nascidos vivos, segundo a idade da mãe}

O total de nascidos vivos no estado de São Paulo diminuiu entre 2000 e 2010. Avaliando-se a tendência segundo a idade da mãe, observa-se que nesseúltimo ano há redução de aproximadamente 117 mil nascimentos para as mães com menos de 30 anos, enquanto entre aquelas com 30 anos e mais ocorre um aumento de 21 mil nascimentos.

Essa tendência diferenciada do número de nascimentos segundo a idade das mães resultou em aumento da percentagem de filhos entre 
as mulheres com 30 anos e mais de idade, que passou de $25,9 \%$, em 2000, para 33,6\%, em 2010.

A distribuição dos nascidos vivos segundo a ordem de filiação reitera o aumento da proporção de mães que tiveram o primeiro filho entre 2000 e 2010 , em um contexto declinante da fecundidade. Enquanto para as mulheres entre 20 e 29 anos tal proporção aumentou de $40 \%$ para $48 \%$, para aquelas entre 30 e 39 anos ela passou de $22 \%$ para $30 \%$. Vale ressaltar, ainda, que entre estas últimas houve incremento também na proporção de segundo filho, de $32 \%$ para $36 \%$, e redução em todas as demais ordens de filiação (Gráfico 7).

\subsection{Fecundidade por coorte}

A análise da fecundidade por coortes mostra que as mulheres que começaram a reprodução entre 15 e 19 anos, em 1960, chegaram ao final do período reprodutivo com uma taxa igual a 3,79 filhos por mulher. Esse valor declina para 3,48, 3,06, 2,73 e 2,44, respectivamente, para as coortes de 1965,1970,1975 e 1980 .

O Gráfico 8 apresenta as coortes completas, entre 1960 e 1980 , e também as incompletas, de 1985 a 2000 . As coortes incompletas foram introduzidas na análise com o intuito de enriquecer o entendimento sobre o comportamento reprodutivo das mulheres paulistas, nesses cinquenta anos, e permitir a realização de inferências sobre tendências futuras. Para todas as coortes, a cúspide da fecundidade ocorre sempre entre as idades de 20 a 24 anos. O segundo momento de intensidade corresponde ao grupo etário de 25 a 29 anos, com exceção das coortes de 1980 e 1995 .

É ilustrativo comentar a coorte de 1960 , porque ela incorpora os diversos momentos da disponibilidade da anticoncepção no estado de São Paulo. De fato, essa coorte apresenta a mais elevada cúspide na faixa etária de 20 a 24 anos, ou seja, em 1965, quando os métodos anticoncepcionais disponíveis eram apenas o coito interrompido, a abstinência e o aborto. E, por outro lado, 6,9\% das mulheres estavam esterilizadas. Já nas idades de 25 a 29 anos, ou seja, em 1970, a fecundidade destas mulheres começa a declinar, e o descenso vai até o final do período reprodutivo, indicando que elas passam a se beneficiar não só de métodos modernos reversíveis como também da esterilização feminina.

De fato, em 1978, 63,9\% das mulheres casadas ou unidas, com idades entre 15 e 44 anos, usavam algum método anticoncepcional, sendo que $27,8 \%$ usavam a pílula e $16,1 \%$ estavam esterilizadas (Nakamura, 1980).

As coortes de 1965 e 1970 apresentam declínio da fecundidade em todas as idades, com exceção do grupo etário 15-19. Enquanto a redução das taxas específicas antes dos 30 anos se mantém em todas as coortes subsequentes analisadas, nota-se que acima dessa idade há 
GRÁFICO 7

Ordem de filiação dos filhos nascidos vivos, segundo idade da mãe

Estado de São Paulo - 2000 e 2010

$\%$

20 a 29 anos

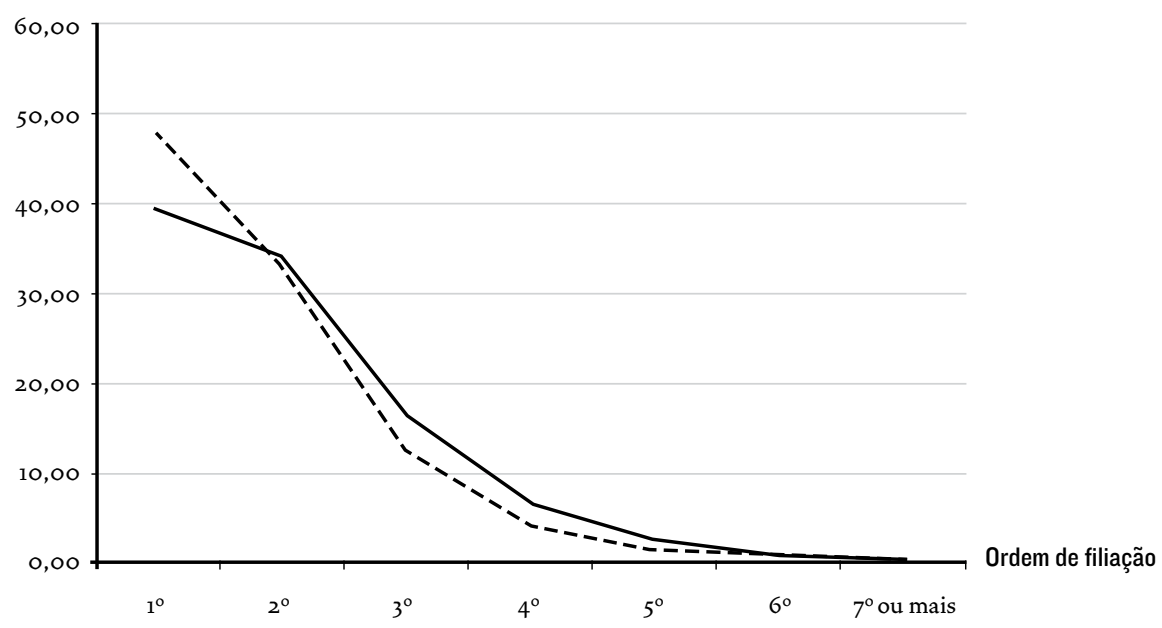

$\%$

30 a 39 anos

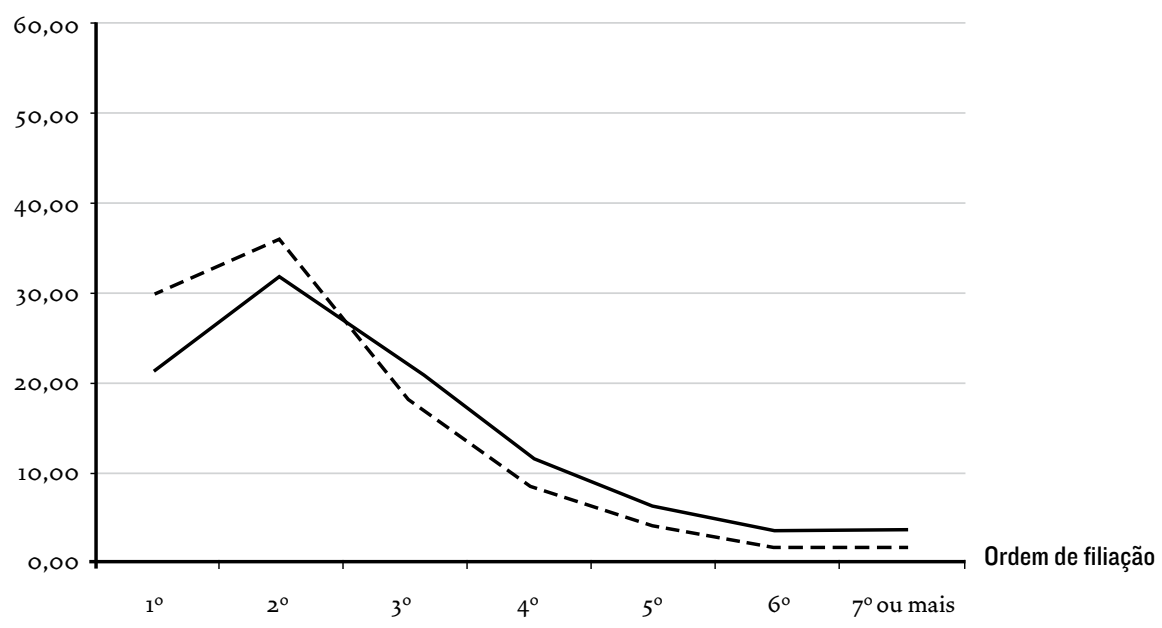

2000

-- - 2010

Fonte: Fundação Seade. 
uma estabilização nas coortes de 1975, 1980 e 1985, exceto no grupo 30-34, quando volta a ocorrer um novo declínio na coorte de 1990.

Esse comportamento influencia a tendência da participação da fecundidade específica das mulheres de 20-29 e 30-39 anos de idade, conforme ilustra o Gráfico 9. Ressalte-se que, na última coorte completa analisada (1980), o grupo 30-39 passou a ter maior participação na taxa de fecundidade total. Considerando-se as coortes incompletas de 1985 e 1990, observa-se também o mesmo fenômeno.

Analisando-se o comportamento da fecundidade por período e coorte, entre 1960 e 1980, observa-se que, a partir do grupo etário 20 a 24 anos, a curva por coorte está sempre abaixo daquela por período (Gráfico 10). Isso se explica pela defasagem de 5, 10, 15, 20, 25 e 30 anos, entre as curvas do período e de coorte, nos grupos etários 20 a 24,25 a 29,30 a 34, 35 a 39, 40 a 44 e 45 a 49 anos, respectivamente.

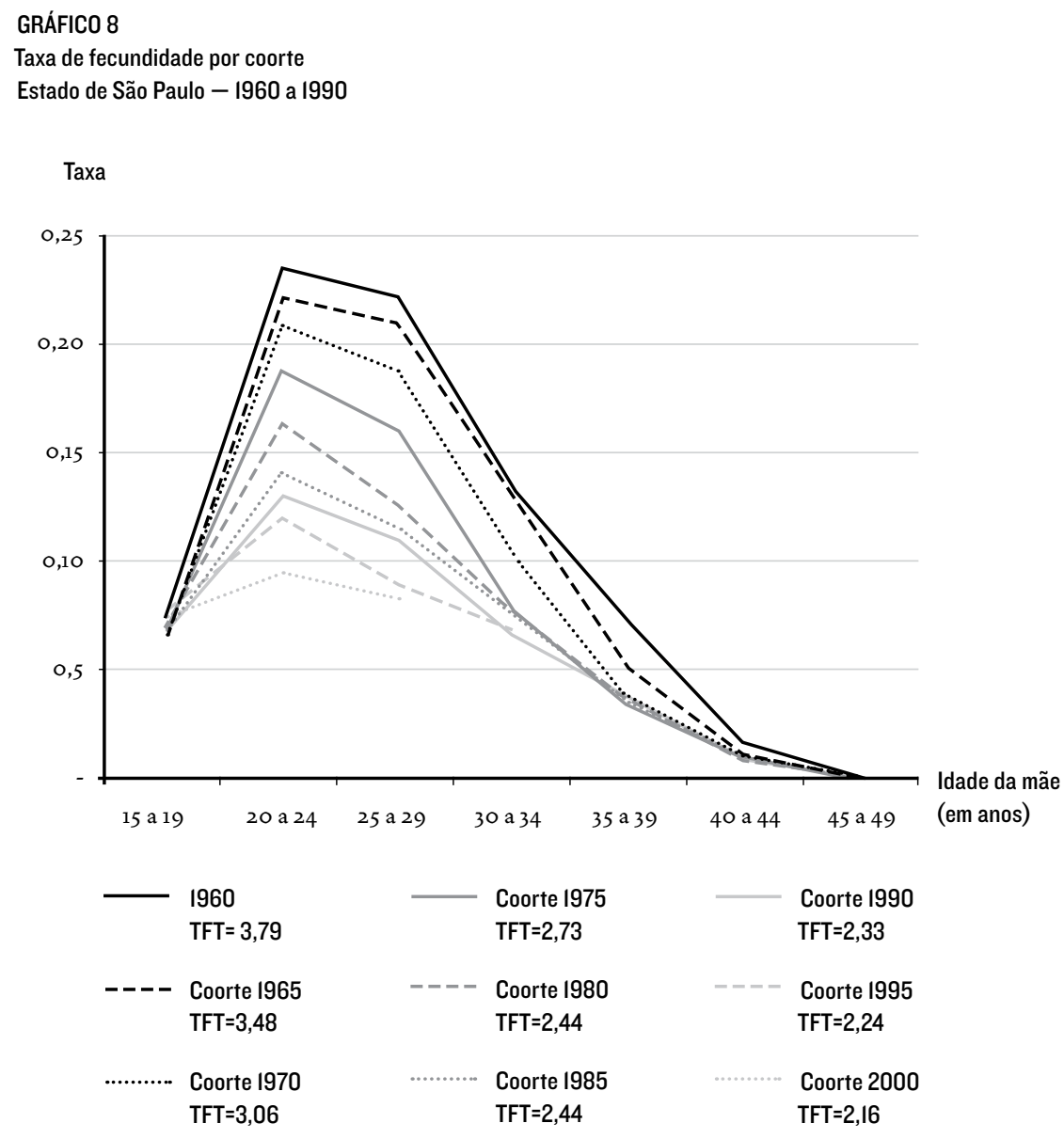




\section{GRÁFICO 9}

Participação da taxa de fecundidade por coorte

Estado de São Paulo - 1960 e 2010

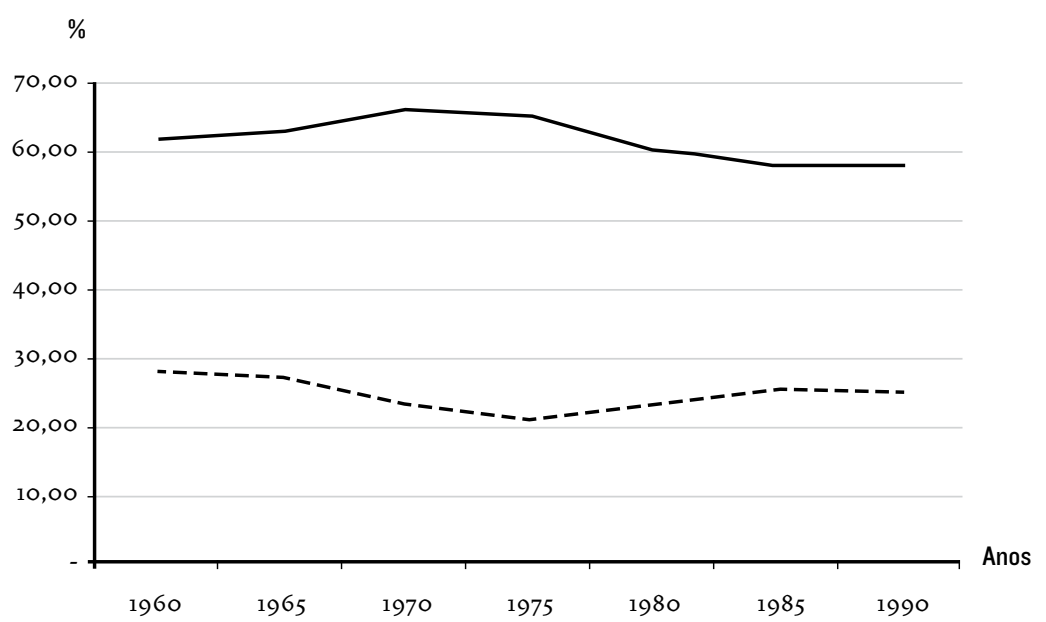

20 a 29

30 a 39

Fonte: Fundação Seade.

Em um regime de fecundidade declinante, essas defasagens levam sempre a valores menores da fecundidade, principalmente nas coortes mais velhas.

\section{CONSIDERAÇõES FINAIS}

A disponibilidade de séries estatísticas do Registro Civil possibilitou a elaboração dos principais indicadores de fecundidade longitudinais (coorte), além dos transversais (período). A análise, assim enriquecida, revelou de forma mais detalhada as tendências e transformações no processo reprodutivo da população paulista.

Entre os resultados encontrados, destacam-se os seguintes.

Em primeiro lugar, confirma-se a queda expressiva dos níveis de fecundidade no período 1960 a 2010 , tanto do ponto de vista dos indicadores transversais como dos longitudinais.

Apesar da tendência de declínio geral da fecundidade, verifica-se um pequeno aumento das taxas específicas para a faixa etária de 30 anos e mais, entre 2005 e 2010 . Observou-se, também, elevação da idade média da fecundidade no contexto das duas abordagens analíticas mencionadas.

Ficou evidente o aumento da participação da fecundidade das mulheres de 30 anos e mais na fecundidade total. Esse fenômeno foi observado tanto na análise por período (1995) como na de coorte(1980). 
GRÁFICO IO

Taxa de fecundidade por idade e por coorte

Estado de São Paulo - 1960 a 1980

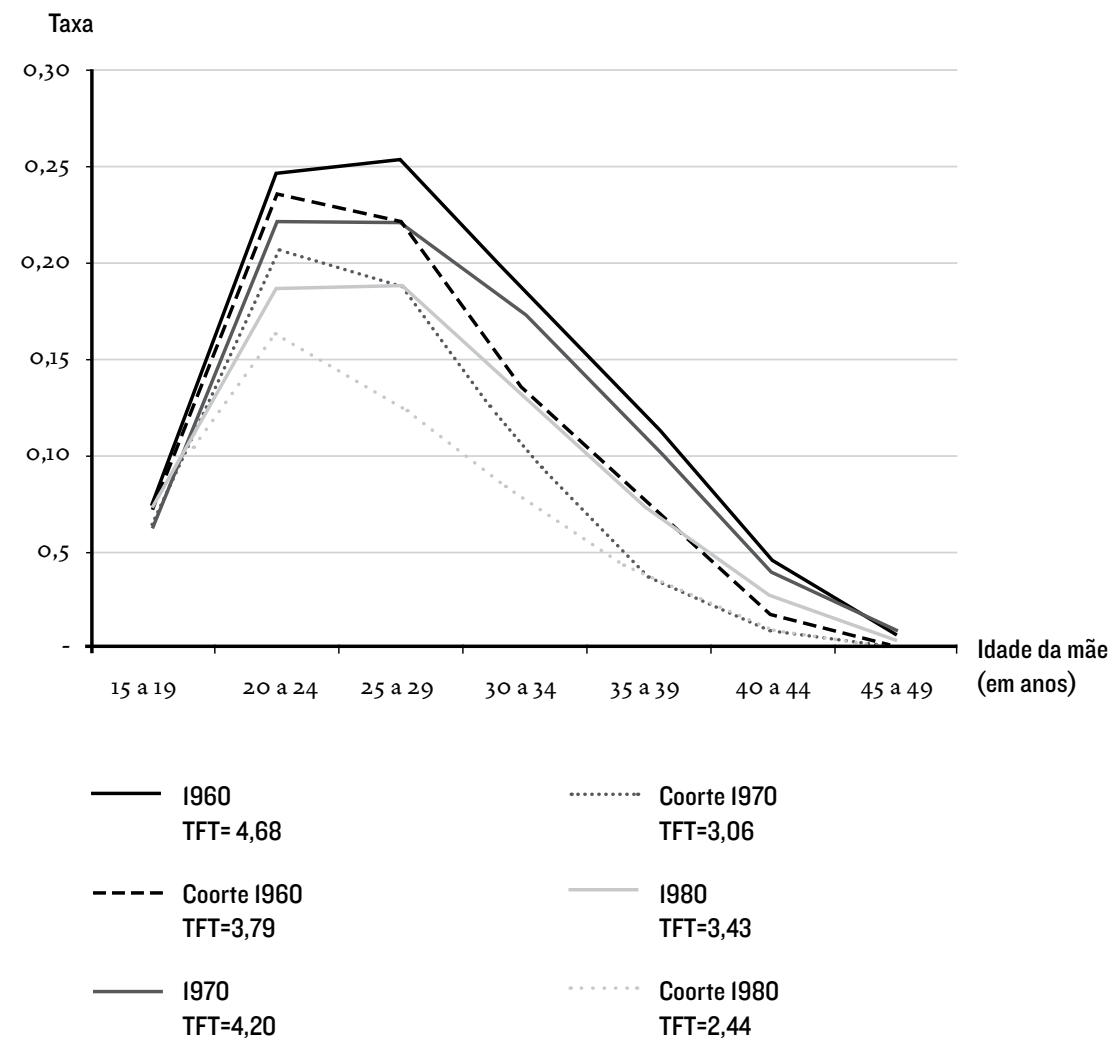

Fonte: Fundação Seade.

Cresceu a proporção de mulheres sem filhos entre 2000 e 2010 em todas as faixas etárias.

Por fim, vale ressaltar a ocorrência de mudanças no padrão etário da fecundidade de período, que apresentou para o ano de 2010 uma curva nitidamente mais dilatada que as anteriores. Esse fenômeno já começa a se delinear no padrão da fecundidade por coorte, considerando-se a tendência observada nas coortes incompletas mais recentes.

Levando em consideração que cresceu o percentual de mulheres sem filhos entre 2000 e 2010 , e que a análise da fecundidade por ordem de parturição revelou o aumento da proporção de nascimentos de primeiros filhos entre as mulheres de 30 a 39 anos de idade, nesse mesmo período, é possível supor a existência de adiamento, temporário ou definitivo, da reprodução da mulher paulista. Tal conclusão suscita novas investigações no sentido de contribuir para o conhecimento do tema. 
Beets, Gisj. "An introduction: late fertility and determinants of postponement behavior". GENUS, Roma: Universitá degli Studi di Roma "La Sapienza", vol. LXIV, n 3-4, julho-dezembro, 2008, pp. 17-31.

Benfam.Pesquisa Nacional sobre Demografiae Saúde, 1996:RelatóriodePesquisa. Rio de Janeiro: Benfam, 1997.

Berquó, Elza \& Garcia, Sandra. "Algumas considerações sobre a reprodução tardia”. In: Turra, Cássio, M. e Cunha, José Marcos P. da (orgs.).População e desenvolvimento em debate: contribuições da Associação Brasileira de Estudos Populacionais. Belo Horizonte: ABEP (Demografia em Debate, vol.4), 2012. 〈http://www.abep.org.br/usuario/GerenciaNavegacao.php?caderno_id $=903$ \&nivel $=1>$.

Billari, Francesco C., Liefbroer, Aart C. \& Philipov, Dimiter (eds.). "The postponement of childbearing in Europe: driving forces and implications". In: Vienna Yearbook of Population Research 2006. Viena:Vienna Institute of Demography, 2006.

Brasil. Ministério da Saúde \& Cebrap. PNDS Saúde da criança e da mulher. PNDS 2006:Dimensões do processo reprodutivo e da saúde da criança.Brasília:Ministério da Saúde/São Paulo:Cebrap, 2009. 〈http:// bvsms.saude.gov.br/bvs/pnds/index.php».

Fundação Seade ("s.f."). Sistema de Estatísticas Vitais, São Paulo. «www. seade.gpov.br>.

IBGE.Censos Demográficos 2010, Rio de Janeiro, 2010. «www.ibge.gov.br». . Censos Demográficos 2000, Rio de Janeiro, 2000. «www. ibge.gov.br>.

Lesthaeghe, Ron J. "The unfolding story of the second demographic transition". Population and Development Review, Nova York, vol.36, $\mathrm{n}^{\circ} 2,2010$.

Lesthaeghe, Ron J. \& Neidert, Lisa. "The second demographic transition in the United States: exception of textbook example?".Population and Development Review, Nova York, vol. 32, n 4, 2006.

Mills, M., Rindfuss, R., McDonald, P. \& te Velde, E. "Why do people postpone parenthood? Reasons and social policy incentives". $\mathrm{Hu}$ man Reproduction Update, Inglaterra, vol. 17, $\mathrm{n}^{\circ}$ 6, 2011.

Nakamura, Milton S., Morris, Leo, Janowitz, Barbara, Anderson, John E. \& Fonseca, Joaquim Barreto. "Contraceptive use and fertility levels in Sao Paulo State, Brazil”. Studies in Family Planning, Nova York, vol. $11, n^{\circ} 7-8,1980$.

Ní Bhrolcháin, Máire \& Beaujouan, Éva. "Fertility postponement is largely due to rising educational enrolment". Population Studies, Londres, vol. 66, nº 3, 2012.

Philipov, Dimiter, Liefbroer, Aart C. \& Billari, Francesco C. (eds.). "The postponement of childbearing in Europe: driving forces 
and implications". In: Vienna Yearbook of Population Research 2006. Viena:Vienna Institute of Demography, 2006.

Sobotka, Thomas, Skirbekk, Vegard \& Philipov, Dimiter. "Economic recession and fertility in the developed world". Population and Development Review, Nova York, vol.37, nº 2, 2011.

Tunalilar, Ozcan \& White, R. "Pathways to childlessness in the United States: a group-based analysis of employment and marital union trajectories". Paper apresentado no PAA 2013, Nova Orleans, 11-13 de abril, na seção Life Course Perspectives on Fertility <http:// paa2013.princeton.edu/abstracts/132522 em 23/04/2013>.

Waldvogel, Bernadette Cunha, Ferreira, Carlos Eugênio Carvalho, Yazaki L. M., Perillo, S. R. \& Aranha, V. J. “Dinâmica demográfica paulista”. Boletim SP Demográfico, São Paulo, ano 12, n $3,2012$.

Waldvogel, Bernadette Cunha y Ferreira, Carlos Eugênio Carvalho. "Estatísticas da vida". São Paulo em Perspectiva, São Paulo, vol. 17, nº 3-4, 2003.

Waldvogel, Bernadette et al. "Projeção da população paulista como instrumento de planejamento". São Paulo em Perspectiva, São Paulo, Vol. $17, n^{\circ} 3-4,2003$.

Wong, Laura Rodriguez \& Perpétuo, Ignes Helena Oliva. (2009) "Uma visão transversal e longitudinal de quatro décadas de queda de fecundidade no Brasil". In: Dimensões do processo reprodutivo e da saúde da criança. Pesquisa Nacional de Demografia e Saúde da Criança e daMulher - PNDS 2006. Brasília: Ministério da Saúde/São Paulo: Cebrap, 2009. 〈http://bvsms.saude.gov.br/bvs/pnds/index.php〉. Yazaki, Lucia Mayumi. "Estatísticas de nascimentos: mães mais velhas e crescimento desenfreado de cesáreas em São Paulo". Boletim SP Demográfico, São Paulo: Fundação Seade, ano 13, n 2, 2013.

. "Fecundidade continua em queda em São Paulo". Boletim SP Demográfico, São Paulo: Fundação Seade, ano 11, n ${ }^{\circ}$, 2011.

Recebido para publicação em 18 de setembro de 2014 .

\section{NOVOS ESTUDOS}

CEBRAP

100 , novembro 2014

pp. 9-25

Elza SAlvatori Berquó é coordenadora do Núcleo de População e Políticas Públicas, Cebrap (popu@cebrap.org.br), e professora emérita da Faculdade de Saúde Pública da Universidade de São Paulo (FSP/USP).

Bernadette Cunha Waldvogel é gerente de Indicadores de Estudos Populacionais da Fundação Sistema Estadual de Análise de Dados — Seade (bvogel@seade.gov.br).

SANDRA GARCIAé pesquisadora do Núcleo de Populaçãoe Políticas Públicas, Cebrap (sandragarcia@ cebrap.org.b), e diretora de Pesquisa da Innovarelab Pesquisa e Consultoria (www.innovarelab.com.br). CARlos Eugenio de CARVAlho Ferreiraéassessor técnico da Fundação Sistema Estadual de Análise de Dados — Seade (ceugenio@seade.gov.br).

TANIA Di GIACOMO DO LAGO épesquisadora do Núcleo de População e Políticas Públicas, Cebrap, e do Instituto de Saúde da Secretaria de Estado da Saúde do Estado de São Paulo e professora assistente do Departamento de Medicina Social da Faculdade de Ciências Médicas da Santa Casa de São Paulo(tania_di@uol.com.br).

LUÍs EDUARDO BATISTA é pesquisador científico, coordenador do Núcleo de Serviços e Sistemas de Saúde do Instituto de Saúde da Secretaria de Estado da Saúde do Estado de São Paulo (lebatista@ saude.sp.gov.br). 